\title{
Vibrations aléatoires dans les circuits industriels
}

\author{
R.J. Gibert CEA - DEMT, Saclay \\ F. Tephany EDF - DER, Chatou \\ P. Thomas EDF - DER, Clamart
}

Les circuits industriels véhiculant des fluides sont souvent le siège de phénomènes vibratoires gênants.

Les zones singulières (pompes, vannes, etc.) de l'écoulement sont en général à l'origine de ces phénomènes, qui peuvent revêtir des caractères assez différents. Les vibrations peuvent être très intenses et conduire à une dégradation rapide des structures. Il s'agit ici d'instabilités résultant d'un couplage fort entre la source d'excitation et la réponse du système. Les vibrations peuvent être d'un niveau plus faible, mais engendrer des ruptures par fatigue ou des usures. Dans ce cas, l'excitation due au fluide peut être caractérisée par des sources aléatoires indépendantes de la réponse.

Les caractéristiques de ces phénomènes peuvent varier notablement selon la nature des fluides (incompressible - compressible) ou celle des structures. On peut observer ainsi des phénomènes dans le domaine des basses fréquences ou des plus hautes fréquences.

L'objet de ce rapport est de rappeler les principaux modèles théoriques de l'excitation aléatoire due aux écoulements instationnaires dans les circuits et de donner un ensemble d'exemples industriels illustrant ses différents aspects.

\section{Random vibrations of an industrial piping system.}

Industrial piping systems conveying liquids are often the source of unwanted vibratory phenomena. The singular zones (pumps, valves, etc.) of the flow are generally speaking the source of these phenomena, which can be of different kinds. The vibrations can be very strong and can lead to structures rapidly deteriorating. This often involves instabilities resulting from a strong coupling between the excitation source and the system response. The vibrations can be of a lower level and can generate fatigue failure and wear. In this instance, the excitation caused by fluid may be characterized by random sources independent of the response.

The characteristics of these phenomena may vary, depending in particular on the type of fluids (compressible or incompressible) or the type of structures. Phenomena can thus be observed in the low or higher frequencies range.

The aim of this report is to briefly describe the main theoretical models of random excitation due to unsteady flows in the piping systems and to give an entire range of industrial examples displaying various aspects.

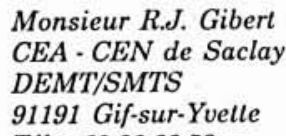

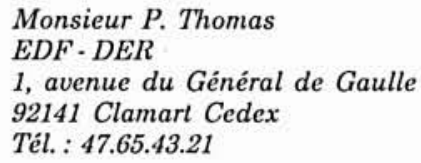

Monsieur F. Tephany $E D F \cdot D E R$

6, quai Watier 78400 Chatou Tél. : 30.71.72.44 


\section{Introduction}

Les vibrations des tuyauteries peuvent avoir 2 origines : mécanique et fluide. Les vibrations d'origine mécanique sont dues principalement aux machines tournantes. Nous n'aborderons pas cet aspect dans le présent exposé. Les vibrations dues au fluide sont induites par l'écoulement au niveau des singularités du circuit. Ces vibrations peuvent contrôler l'excitation fluide et conduire à l'instabilité du système (couplage fort). Elles peuvent également traduire la réponse du système au bruit engendré par les singularités de l'écoulement (couplage acoustique-mécanique).

Dans le cas du couplage fort, ce sont bien souvent les paramètres locaux de la structure et de l'écoulement (fréquence réduite, nombre de Strouhal, corrélation spatiale) qui déterminent l'instabilité : citons les travaux consacrés aux excitations par les tourbillons de Karman, en particulier les phénomènes d'accrochage observés sur un cylindre oscillant rapportés entre autres dans un document de synthèse publié par la SHF [réf. 1]; citons également les travaux concernant les instabilités hydroélastiques des écoulements longitudinaux [réf. 2], les analyses sur le comportement des faisceaux de tubes dans les échangeurs [réf. 3] et aussi les études portant sur le flottement des ailettes de turbomachine [réf. 4 et 8]. Nous laisserons de côté dans la suite de l'exposé tous ces mécanismes d'instabilité à caractère local, pour nous intéresser plus spécialement à l'excitation à distance liée à la propagation d'ondes acoustiques dans un circuit.

On distingue le cas des circuits hydrauliques et celui des circuits vapeur, le premier concernant essentiellement les vibrations à basse fréquence, à faible Mach, le second mettant en jeu des déformations de coque à des fréquences plus élevées.

Trois exemples figurant dans l'exposé illustrent les aspects importants et les difficultés rencontrées tant au niveau expérimental qu'au niveau de la modélisation.

\section{Sources vibratoires associées aux singu- larités d'écoulement des circuits hydrau- liques}

\subsection{Singularités passives [référence 7]}

L'écoulement dans les circuits industriels est généralement turbulent; le régime d'écoulement stable n'est atteint que sur la base d'un taux de fluctuations fini stationnaire. Mais les fluctuations n'ont pas partout la même intensité: la turbulence établie dans un tuyau droit a un spectre beaucoup plus étalé et d'un niveau bien moindre que celle attachée à certaines zones singulières des circuits - élargissement, coudes, vannes dont l'énergie est essentiellement située aux basses fréquences.

C'est à l'endroit de ces singularités, où la turbulence est plus intense, qu'on situe les sources de vibrations de la tuyauterie. On considère que les fluctuations imputa- bles à la compressibilité du fluide et au mouvement des parois n'affectent pas le régime d'écoulement instationnaire, de sorte que la source de vibrations qui lui est associée peut être caractérisée indépendamment de la réponse du système couplé fluide-structure.

Pour expliciter cette source, on décompose la vitesse de l'écoulement en ses 3 contributions permanente, turbulente et acoustique-mécaniques, ces 2 dernières restant petites devant la première. Fluctuations de pression et de masse volumique sont liées par la relation de compressibilité. La linéarisation des équations de Navie-Stokes par rapport aux fluctuations de vitesses acoustique-mécanique aboutit à une équation de propagation où le second membre représente la source d'excitation due à l'écoulement instationnaire.

L'intégration des termes sources porte sur la seule zone singulière de l'écoulement caractérisée par des fluctuations plus intenses. La surface délimitant cette zone est composée des parois $\Sigma$ de la structure de confinement et des sections $\mathrm{S}$ d'adhérence au fluide non perturbé. En appliquant la formule de Green à cette intégrale de volume $v$, on fait apparaître 3 termes :

- une source acoustique liée à la compressibilité du fluide dans le volume;

- une source aux limites $S$ du fluide perturbé;

- une source d'excitation directe de la structure $\Sigma$ par les fluctuations de pression locale.

En pratique, dans le cas de structures souples et en présence d'un fluide très peu compressible (plaques ou coques minces pour un écoulement d'eau), on peut négliger les 2 premiers termes et ne considérer que les modes de vibrations locaux (qui ne font pas intervenir les effets à longue distance).

C'est en revanche l'hypothèse inverse qui domine dans le cas des problèmes purement acoustiques, parce qu'on considère essentiellement les modes liés au fluide compressible, l'excitation locale de la paroi ne jouant plus qu'un rôle secondaire.

Les fluctuations turbulentes engendrées par les singularités hydrauliques sont caractérisées par une concentration d'énergie vers les basses fréquences; ainsi les longueurs d'ondes associées aux phénomènes acoustiques imputables à ces fluctuations sont généralement grandes devant le diamètre de la tuyauterie (du moins tant que le nombre de Mach demeure faible); alors la propagation dans les tuyauteries s'effectue par ondes planes. Par ailleurs, ce sont les mouvements de flexion d'ensemble de la tuyauterie qui sont les plus excités. C'est par conséquent sur les premiers modes acoustique-mécaniques que l'on va projeter le champ de pression instationnaire associé aux singularités. Or l'étendue de la zone occupée par les fluctuations reste toujours assez faible (quelques diamètres au maximum), ce qui permet de considérer que le déplacement modal de la tuyauterie demeure constant dans le volume d'intégration. Pour la même raison, on peut regarder constante la variable de pression acoustique dans cette zone, mais non son gradient qui subit une discontinuité du fait du mouvement de la tuyauterie. Moyennant cette analyse, on fait apparaître 2 termes $\Delta p$ et $\Delta q$ dans l'expression de la source acoustique, qui correspondent à des discontinuités de la pression et du débit-masse acoustique. 
Finalement la représentation de la source vibratoire sous la forme $(\Delta p(t), \Delta q(t))$ de discontinuités de la pression et du débit acoustique est corrélative de la représentation du système dans lequel elle se propage (propagation en ondes planes dans une structure de confinement élancée). Le choix d'une telle représentation pour le système lui-même privilégie le rôle souvent critique des vibrations dans le domaine des basses fréquences.

Il est désormais profitable d'étudier l'allure des densités spectrales des fonctions $\Delta p(t)$ et $\Delta q(t)$, et d'essayer à partir d'une analyse dimensionnelle de définir un certain nombre de leurs grandeurs physiques caractéristiques. En variables adimensionnelles définies par rapport à des grandeurs de référence $V_{0}, P_{0}$ et $L$, les fluctuations instationnaires sont caractérisées par $v / V_{0}$ et $p / \rho_{0} V_{0}^{2}$ qui sont fonctions de $\vec{r} / L$ et $V_{0} t / L$. On peut montrer que les fluctuations instationnaires dans le domaine des basses fréquences sont peu dépendantes des nombres de nombre de Reynolds $R e=V_{0} L / v$ et de Mach $M=V_{0} / C$ de l'écoulement.

Les spectres adimensionnels des champs de pression turbulente $p_{t}$ aux parois de la singularité s'expriment en fonction de la fréquence adimensionnelle $s=f L / V_{0}$ (nombre de Strouhal). Ils ont une allure de type turbulent, décroissant en fréquence, à large bande. Les discontinuités de la fluctuation de la pression et du débit acoustique, $\Delta p(t)$ et $\Delta q(t)$ sont des fonctions linéaires des champs $p_{t}(\vec{r}, t)$ : leurs spectres adimensionnels ont l'allure indiquée à la figure 1 .

L'analyse expérimentale effectuée sur un élargissement brusque confirme l'ensemble des points que l'on vient d'évoquer. En ce qui concerne la zone instationnaire locale, on distingue 3 domaines: celui des fluctuations de pression à très basse fréquence $(s<0,01)$ situé à l'aval immédiat de l'élargissement dans la poche d'écoulement décollé, celui des fluctuations très intenses ( $10 \%$ de la perte de charge environ) dans une gamme de fréquence supérieure $(s \simeq 0,2)$ situé dans la zone de recollement du jet, enfin plus en aval, celui de la turbulence établie classique dans une tuyauterie. La longueur de référence est la différence des 2 diamètres; elle caractérise la dimension transversale de la poche décollée : si l'on rapporte la distance à l'aval de l'élargissement à $(D-d)$, on constate que l'évolution de la pression statique est indépendante du rapport d'évasement $d / D$. La vitesse de référence est celle de l'écoulement dans le tuyauterie amont (qui vaut celle du jet).

En amont, et loin à l'aval de la singularité, on mesure des fluctuations de nature acoustique (ondes planes). Elles représentent la réponse du circuit aux sources créées par l'élargissement: l'examen suivant la gamme des fréquences du déphasage entre les signaux délivrés par 2 capteurs situés de part et d'autre de la singularité dénote un comportement différent selon qu'il s'agit d'une discontinuité de pression ou de débit acoustique. On utilise cette propriété pour connaître la nature de la source. L'identification des densités spectrales $S_{\Delta p}$ et $S_{\Delta q}$ suppose un certain nombre de précautions expérimentales, et le concours d'outils théoriques permettant de connaître les fonctions de transfert $G_{\Delta p}$ et $G_{\Delta q}$ entre point source et point d'observation (modèle de propagation en ondes planes) car on ne mesure pas la source

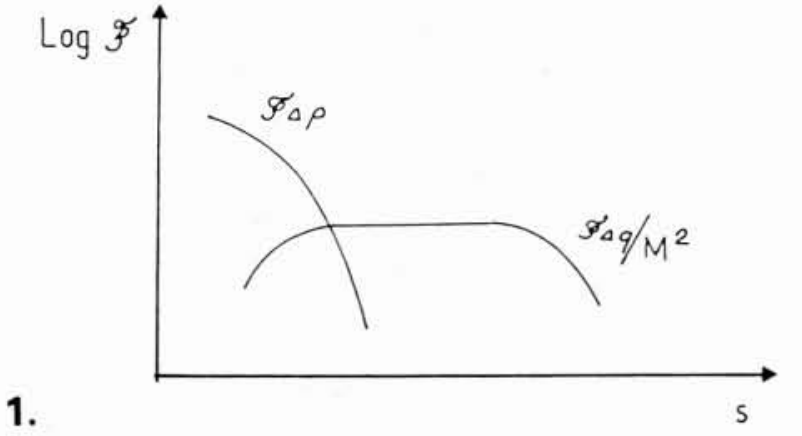

acoustique, mais la réponse du circuit à cette source. La figure 2 (page suiv.) illustre l'ajustement de ces spectres; y figurent les densités spectrales des sources ajustées, les densités spectrales brutes des 2 capteurs amont et aval et les densités spectrales de ces 2 mêmes capteurs obtenues par la formule:

$$
S=\left|G_{\Delta p}\right|^{2} S_{\Delta p}+\left|G_{\Delta q}\right|^{2} S_{\Delta q}
$$

Ces dernières courbes doivent coïncider si l'ajustement est correct.

En utilisant la représentation adimensionnelle suivante :

$$
\begin{aligned}
& F_{\Delta p}=\frac{S_{\Delta p}}{\Delta p^{2}} \frac{V_{0}}{D-d} \\
& F_{\Delta p}=\frac{S_{\Delta q}}{\Delta p^{2}} \frac{V_{0}}{D-d}\left[\frac{C}{(D-d)^{2}}\right]^{2}
\end{aligned}
$$

On obtient les allures caractéristiques de la figure 3 (page suiv.).

Ainsi, pour la turbulence locale engendrée par les singularités :

- l'analyse dimensionnelle fondée sur le choix approprié des grandeurs permanentes de référence, permet de caractériser les fluctuations locales engendrées par les singularités;

- en particulier, les caractéristiques spectrales et interspectrales des fluctuations peuvent s'exprimer en fonction de variables adimensionnelles suivant des lois standard pour chaque singularité. Cela permet de constituer un formulaire directement utilisable pour un projet d'installation industrielle;

- ces lois standards sont pratiquement indépendantes des nombres de Reynolds et de Mach;

- une représentation du mécanisme d'instabilité de l'écoulement permet d'orienter le choix des grandeurs de référence : dimensions caractéristiques, pression et vitesse de référence qui sont souvent en pratique la recompression et la vitesse maximum moyenne dans la singularité;

- l'écart-type des fluctuations dépend de la position du capteur et passe par un maximum d'environ 0,1 à 0,2 fois la perte de charge;

- la densité spectrale adimensionnelle des fluctuations suit une forme décroissante de type "Dryden "; le nombre de Strouhal de coupure est de l'ordre de 0,2 à 0,5 dans la zone où les fluctuations sont maximum. 
2.
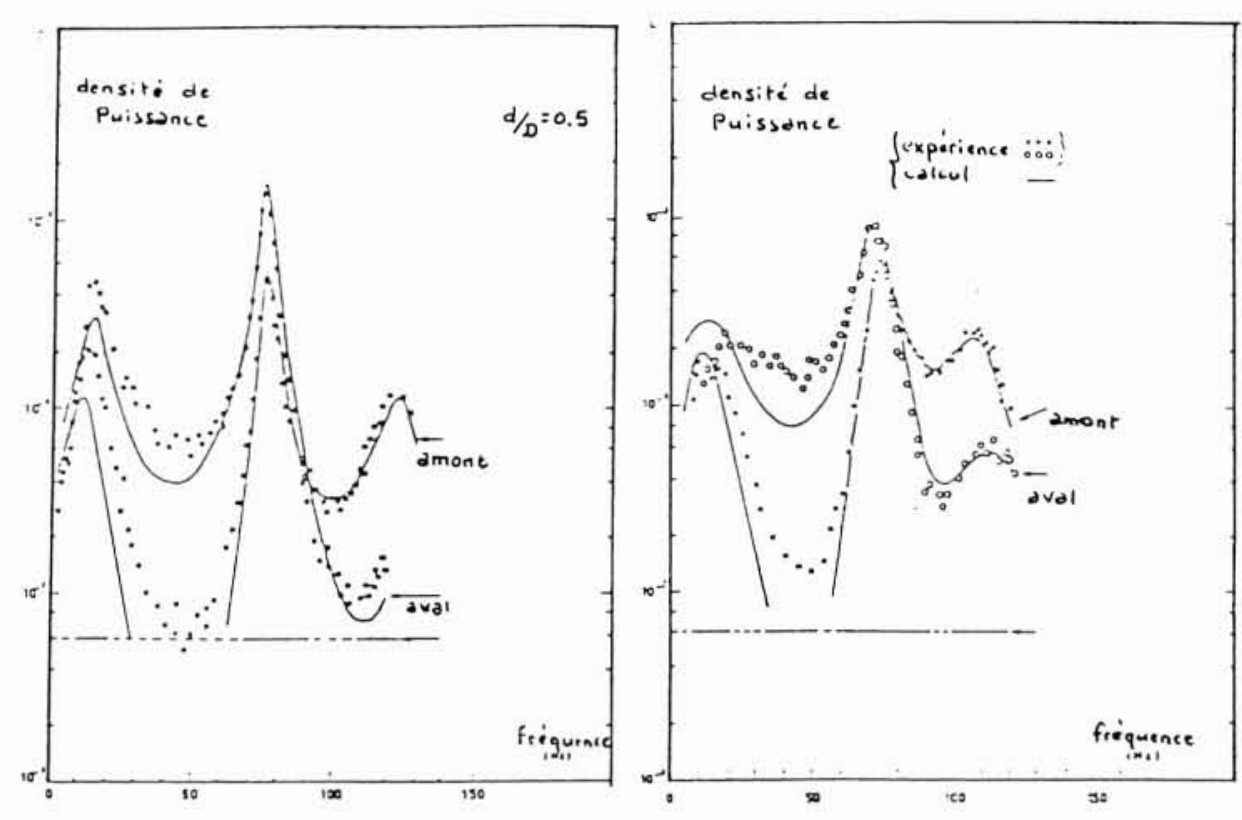

3.
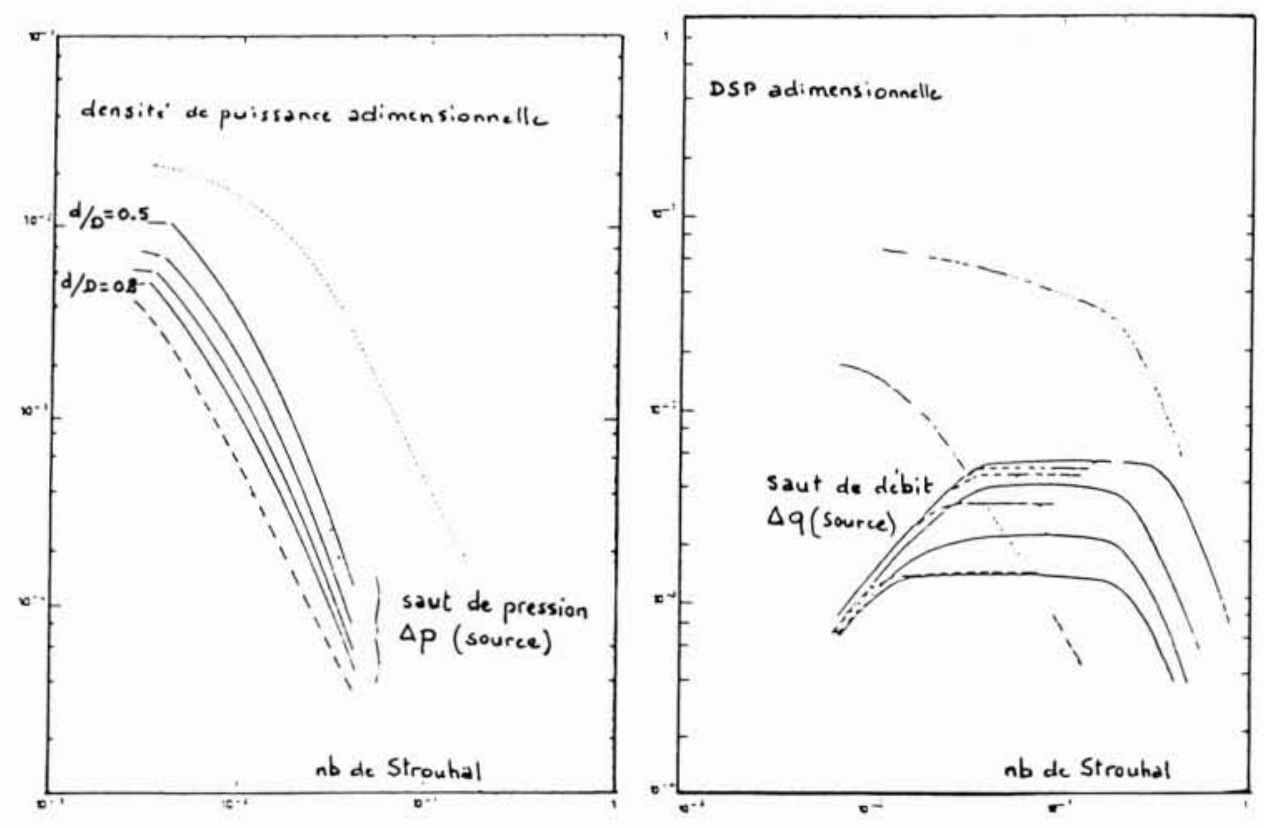

Ainsi pour la source acoustique associée à cette turbulence :

- le nombre de Strouhal de coupure est en général inférieur à celui des fluctuations locales. Même en adoptant un majorant des fréquences d'émission correspondant à une fréquence adimensionnelle de 0,5 , on demeure bien en-dessous de la fréquence de coupure des circuits. On vérifie par là que la propagation s'effectue par ondes planes;
- le problème de la réponse du circuit aux sollicitations d'une source représentée par une discontinuité de la pression et du débit acoustique est complètement monodimensionnel;

- la singularité se comporte comme une source de pression acoustique aux faibles nombres de Strouhal et comme une source de débit acoustique aux nombres de Strouhal plus élevés quand le nombre de Mach n'est pas trop faible, les spectres de ces 2 discontinuités étant pratiquement disjoints. 


\subsection{Pompes}

L'analyse présentée dans le paragraphe précédent vaut aussi dans une large mesure pour l'organe de circulation, si l'on cherche à le caractériser par une source acoustique se propageant en ondes planes dans le reste du circuit: la représentation de cette source en termes de discontinuité de pression et de débit reste en effet possible dans la mesure où il y a indépendance entre la source et la réponse du circuit. Cependant, l'identification expérimentale des termes sources est plus délicate que dans le cas des singularités passives: on a vu que pour ces dernières, une parfaite connaissance des fonctions de transfert et des impédances aux limites du circuit sont nécessaires pour l'ajustement des spectres d'autocorrélation des sources, et que dans certains cas (par exemple l'élargissement brusque) il faut considérer l'impédance acoustique associée à la zone singulière. Or s'il existe des modèles d'impédance quasi statiques convenables pour les différents composants des circuits, il reste en revanche à perfectionner ceux qui décrivent la pompe.

Une autre difficulté apparaît à l'examen du spectre de pression fluctuante engendrée par la pompe: de nombreuses raies discrètes liées à la rotation se superposent au bruit large bande engendré par la turbulence. Par suite, les pics existant dans les spectres de réponse du circuit, ne correspondent plus nécessairement à des résonances acoustique-mécaniques, ce qui complique l'analyse des fonctions de transfert. En outre, la coïncidence entre une raie de pompe et une résonance acoustique-mécanique peut conduire à une interaction entre l'excitation due à la pompe et la réponse du circuit.

D'un autre côté, la modélisation analytique des termes sources est elle aussi plus délicate que dans le cas des sources passives : en effet, les mécanismes des instationnarités de l'écoulement sont nombreux et complexes, ce qui rend difficile le choix de grandeurs caractérisant la pompe dans son ensemble. On peut bien sûr tenter de caractériser chacun des facteurs qui contribuent à la génération du bruit : bruit de raie, provenant des interactions roue-diffuseur-volute, interception des sillages par les aubages du diffuseur ou par le bec de volute, asymétrie de l'écoulement, qui forment le bruit à caractère périodique que l'on retrouve à la fréquence de rotation, à la fréquence de passage des aubes, et sur leurs harmoniques, et bruit large bande, provenant des fluctuations du débit-masse, turbulence d'entrée de roue, turbulence de sillage et de jet, instationnarités auto-induites par l'écoulement sur les parties mécaniques (hydroélasticité), voire recirculations dans le cas des régimes de fonctionnement à débit partiel.

Mais si on attache à chacune de ces contributions un modèle de source (par exemple pour le bruit de circulation, un dipôle acoustique compact d'intensité égale à la variation de portance de l'aube), on est confronté au problème de la connaissance du champ de propagation dans le corps de pompe. On peut envisager d'appliquer certaines méthodes expérimentales comme la technique de réciprocité qui consiste à inverser point source et point d'observation dans la roue de pompe et sur la tuyauterie afin d'identifier les coefficients de leur fonction de transfert.
Un travail important a été réalisé voici une vingtaine d'années par Simpson et Clark [réf. 5] et repris récemment par Imaichi [réf. 6] pour déterminer le bruit hydraulique produit au refoulement d'une pompe à basse vitesse spécifique. Constatant que le bruit de raie est prépondérant dans ce cas, ils considèrent que 2 mécanismes contribuent à la production de ce bruit: les intéractions à potentiel entre parties fixes et mobiles d'une part, et le sillage visqueux issu des aubages de la roue d'autre part. On sait établir pour chacune de ces contributions les expressions des fluctuations de vitesse et de pression à la fréquence de passage des aubes. Celle correspondant aux interactions entre parties fixes et mobiles est fondée sur la théorie du fluide parfait. Celle correspondant à l'interception des sillages visqueux est fondée sur le modèle de Sears. On aboutit finalement au niveau de bruit global défini au refoulement de la pompe dont l'expression est bien connue :

$$
S_{p}=20 \log _{10}\left[\frac{\alpha}{S} \frac{\Delta P_{0} Q / b}{\Omega a^{2}}\right]
$$

où $S$ est la vitesse spécifique, $b$ la largeur de sortie de roue, $Q$ le débit et $\Delta P_{0}$ la hauteur de la pompe, $\Omega$ sa vitesse de rotation et $a$ le rayon moyen de l'aubage. $\alpha$ est un coefficient multiplicateur dépendant des unités dans lesquelles on exprime les grandeurs précédentes [cf. référence 5].

Il est clair qu'à ce stade, on n'a pas caractérisé la pompe en tant que source acoustique comme cela a été fait pour les singularités passives.

\section{Réponse acoustique-mécanique des cir- cuits}

\subsection{Modélisation dans le domaine des basses fréquences}

Le calcul des vibrations résultant des efforts fluctuants engendrés par les sources associées aux singularités du circuit et s'exerçant sur les parois des tuyauteries nécessite de déterminer les caractéristiques dynamiques du système couplé fluide-structure. Dans le cas de l'eau, les longueurs d'ondes acoustiques des vibrations sont très grandes devant les dimensions transversales de tuyaux. Les vibrations des tuyaux sont telles que leur section droite ne se déforme pas. On représente donc la tuyauterie par des éléments de poutre, et le fluide par son équation de propagation en ondes planes, le couplage s'opérant par effet de fond au niveau des singularités. Le problème acoustique-mécanique est monodimensionnel et le système des équations locales couplées pour un élément de tube de rayon de courbure $R(s)$ et de section $S(s)$ s'écrit :

$$
\begin{aligned}
& M \overrightarrow{\ddot{\delta}}+\rho S \overrightarrow{\ddot{\delta}} t+K \vec{\delta}-\frac{P}{R} S \vec{t}+\rho \frac{\delta \Sigma}{d s} \vec{\ell}=0 \\
& \begin{aligned}
\frac{\partial}{\partial s}\left(S \frac{\partial P}{\partial s}\right)-\frac{S}{C^{2}} \frac{\partial^{2} P}{\partial t^{2}} & \overrightarrow{\ddot{\delta}} \cdot \vec{t} \\
& -\rho S \frac{d S}{R} \overrightarrow{\ddot{\delta}} \cdot \vec{\ell}=0
\end{aligned}
\end{aligned}
$$


$\delta$ est le déplacement de la section droite, $M$ et $K$ sont les opérateurs de masse et de raideur du tube sans fluide, $P$ la pression acoustique sur une section droite $S$ et $C$ la célérité du son dans le fluide (figure 4).

4.

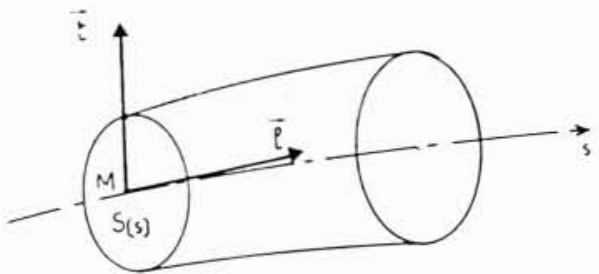

Les singularités apparaissant dans les termes de coulage des équations ci-dessus sont les variations de section et les changements de direction. Ces termes de couplage s'apparentent à une force fluctuante appliquée à la conduite et à un débit fluctuant imposé au fluide.

\subsection{Modélisation dans le domaine des moyen- nes fréquences}

La représentation ci-dessus du couplage acoustiquemécanique n'est légitime que dans le domaine des basses fréquences qui sont pour nous les fréquences inférieures à la première résonance de la tuyauterie en déformation de coque (telle que: $\omega D^{2} / C^{\prime} h \ll 1$ ) et à la première résonance acoustique transverse clans une section (telle que $\omega D / C<1$ ) où $D$ est le diamètre de la tuyauterie, $h$ son épaisseur, $C$ et $C^{\prime}$ les célérités des ondes dans le fluide et dans le métal.

Or, pour certaines lignes industrielles véhiculant des fluides à grande vitesse, le spectre d'excitation des sources acoustiques peut déborder le domaine basse fréquence. Il faut dans ce cas disposer d'un modèle valable sur toute la gamme d'excitation, comportant donc la représentation des déformations de coque et des ondes transverses de fluide.

$\mathrm{Si}$ on se limite au cadre de l'analyse vibratoire linéaire, le modèle le plus simple permettant de résoudre le problème de la propagation monodimensionnelle des ondes de coque et des ondes de pression est basé sur l'utilisation des matrices de transfert. Cependant, si l'on veut prendre en compte le couplage acoustique-mécanique, la détermination des matrices élémentaires est alors délicate. Par contre, l'analyse numérique par éléments finis ne pose pas de problème théorique, mais elle nécessite la manipulation de gros modèles, comme on le verra dans les applications. Ces modèles peuvent devenir très lourds si l'on veut en plus prendre en compte le fluide interne. Un bon compromis consiste à utiliser des éléments finis simplifiés comportant les solutions stationnaires de la coque et du fluide dans la section du tube, ainsi que les termes de couplage acoustique-mécanique, et de résoudre le problème de propagation suivant l'axe des tuyauteries (comme dans le cas des basses fréquences). Pour la coque, les fonctions de forme retenues sont les harmoniques de Fourier, de même que pour la variation circonférentielle de la pression. La variation radiale de la pression se décompose sur des fonctions de Bessel. En définitive, on utilise un élément de poutre à deux nœuds, chaque nœud supportant une centaine de degrés de liberté. On peut noter que pour les applications basse fréquence, il suffit de conserver un très petit nombre d'harmoniques qui permettront de représenter des phénomènes correctifs tels que l'effet Poisson (dilatation de la section dans son plan) ou la compressibilité transverse du fluide dans les coudes, qui interviennent d'autant plus qu'on approche les fréquences de coupure correspondantes.

Les développements en cours visent principalement à caractériser correctement l'effet tridimensionnel des coudes aux moyennes fréquences. C'est pourquoi on a entrepris la détermination des coefficients de couplage entre les différents harmoniques de Fourier et de Bessel intervenant dans un coude torique. On s'attend en particulier à trouver un couplage significatif entre les harmoniques 0,1 et 2 de la coque et du fluide.

Les figures 5 et 6 illustrent par exemple le cas d'une conduite rigide comportant un coude 1,5 à 90 degrés, et à l'extrémité de laquelle on injecte en onde plane. L'onde plane génère dans le coude une onde transverse qui ne se propage pas dans la ligne.

On note par contre que la composante transversale se propage hors du coude lorsqu'on entre dans le domaine des moyennes fréquences (cf. figure 7, sur laquelle on voit en outre apparaitre une composante d'ovalisation).

\section{Applications industrielles}

L'analyse numérique des problèmes posés par les vibrations des lignes de tuyauteries en écoulement permanent (en général, ce sont des problèmes de fatigue à grand nombre de cycles) se décompose en plusieurs étapes :

- identification de la source acoustique responsable des vibrations;

- calcul des caractéristiques dynamiques du système couplé fluide-structure;

- utilisation de la fonction de transfert entre la source acoustique et une contrainte d'endommagement, pour calculer les paramètres statistiques de celle-ci en fonction de ceux de l'excitation fluide.

\subsection{Réponse d'un circuit à une source acousti- que}

On va illustrer la mise en œuvre d'un modèle d'ondes planes pour l'identification expérimentale d'une singularité, et le calcul de la réponse acoustique-mécanique d'une ligne de tuyauterie.

\section{La boucle d'essai}

Pour caractériser une pompe en tant que source acoustique au sein d'un circuit industriel, un programme exéprimental a été engagé à E.D.F. sur la boucle Everest de St. Denis. Cette boucle (figure 8) comporte une pompe $(\mathrm{P})$, un by-pass (B) et plusieurs vannes (V), de sorte que l'on peut obtenir des configurations d'écoulement variées. La veine en eau présente en gros trois diamètres 

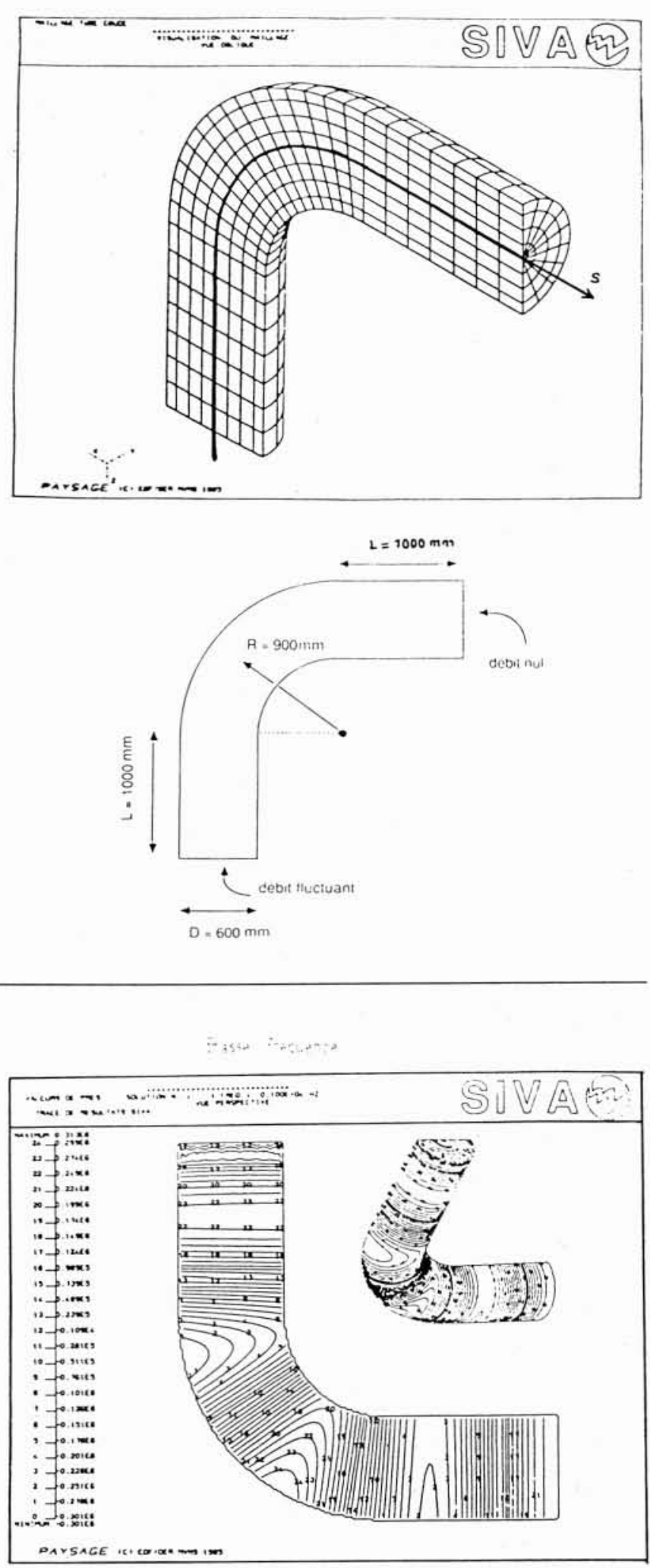

Moyenne fréquence

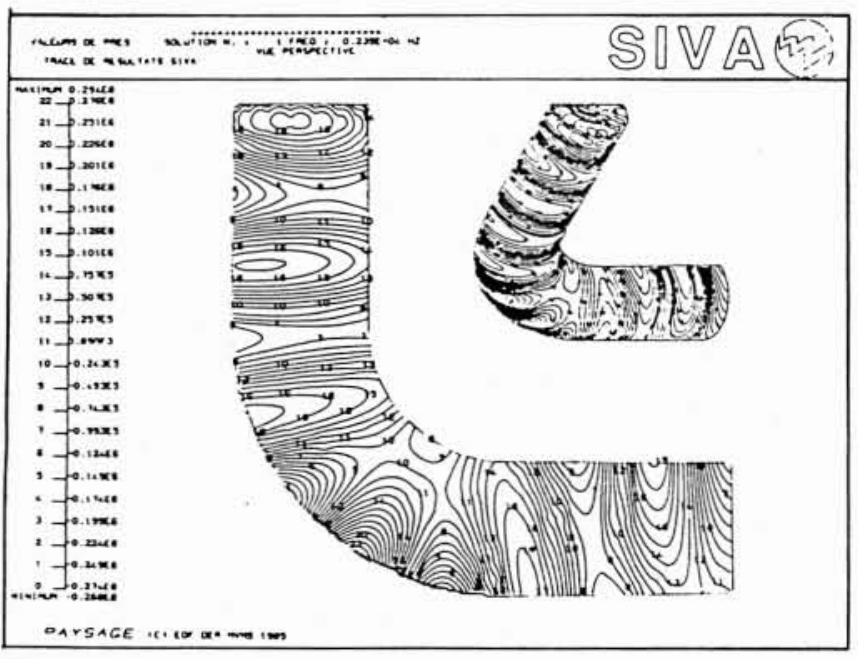

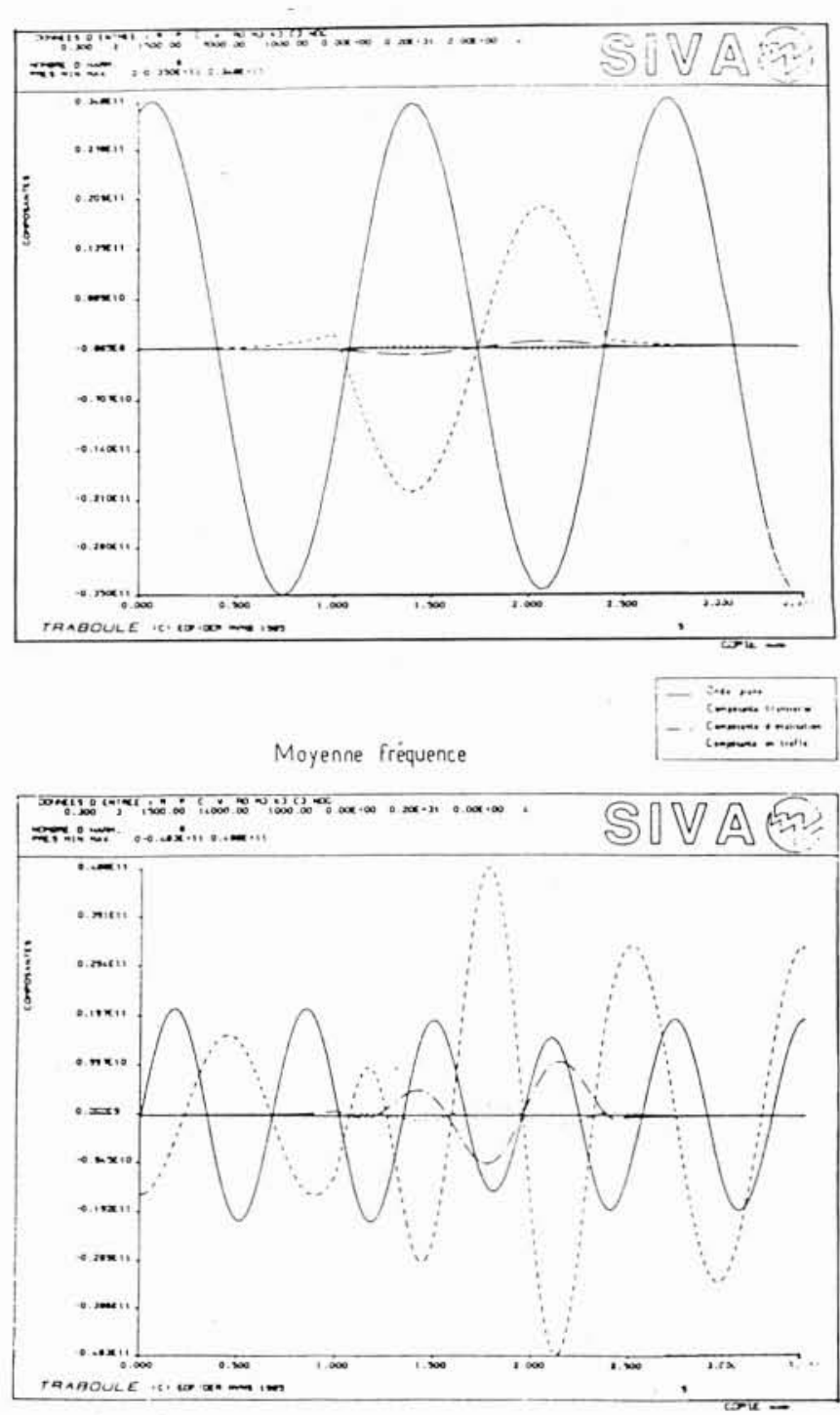

7.

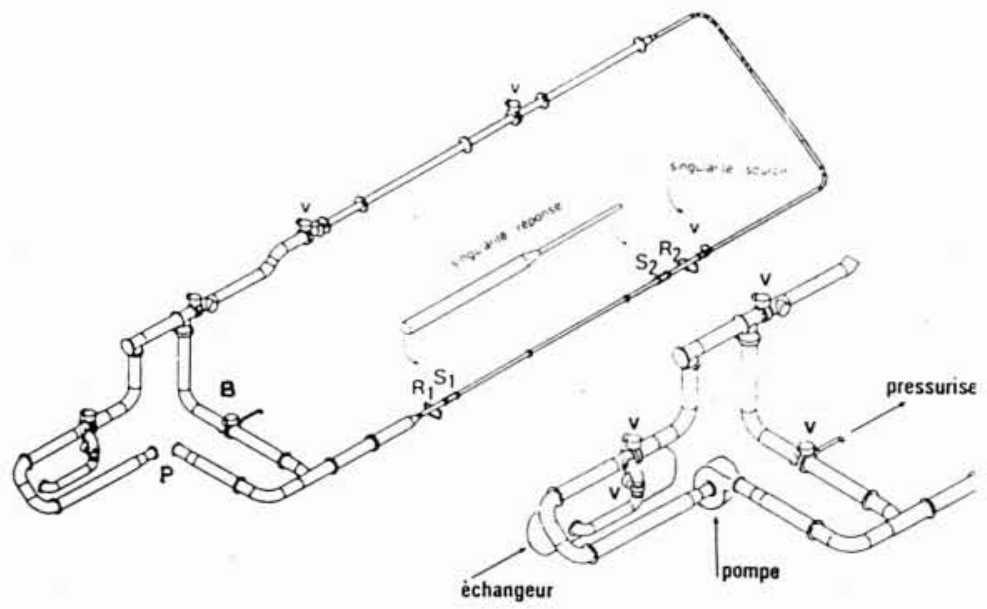

8.

5. Effet tridimensionnel d'un coude acoustique.

6. Modélisation tridimensionnelle.

7. Modèle de poutre.

8. Banc d'essai sur boucle Everest 
9.

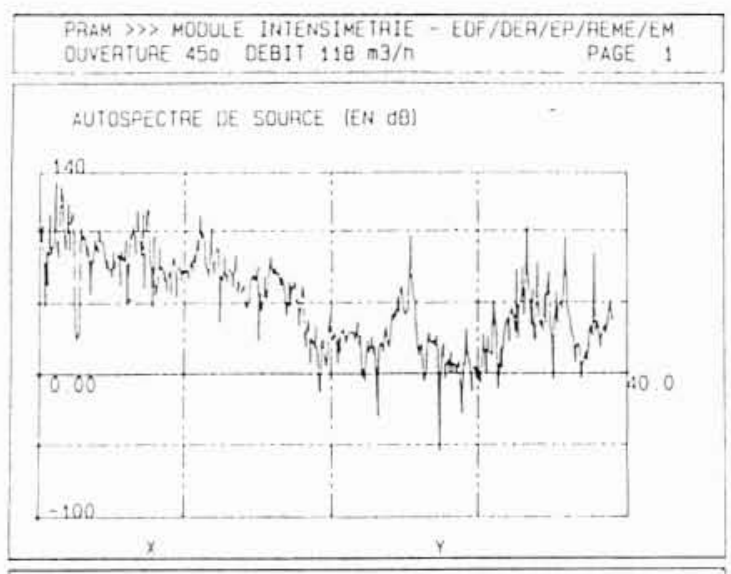

COMERENCE 4-: (T.: 21

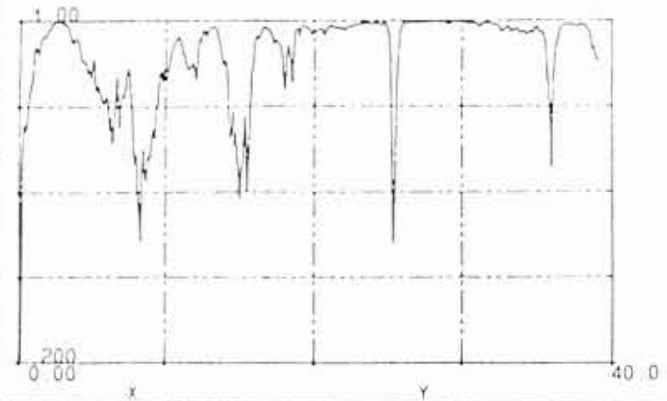

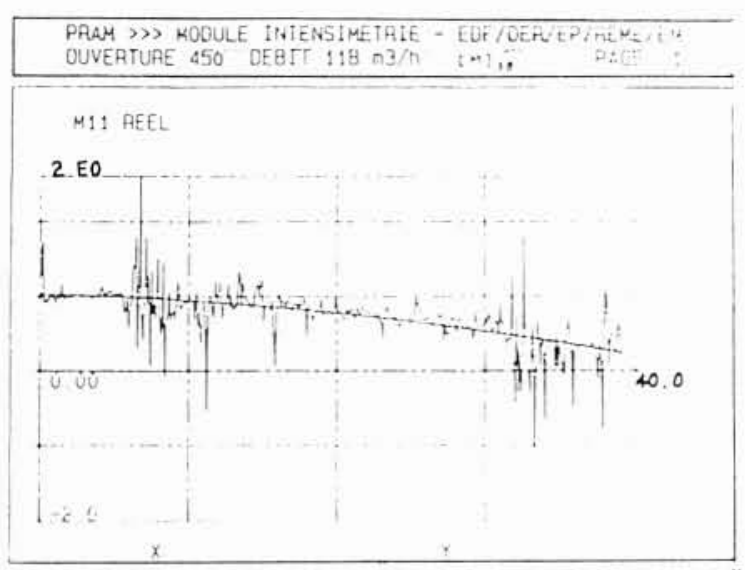

M:1 IMAGINATAE

2.30

10.

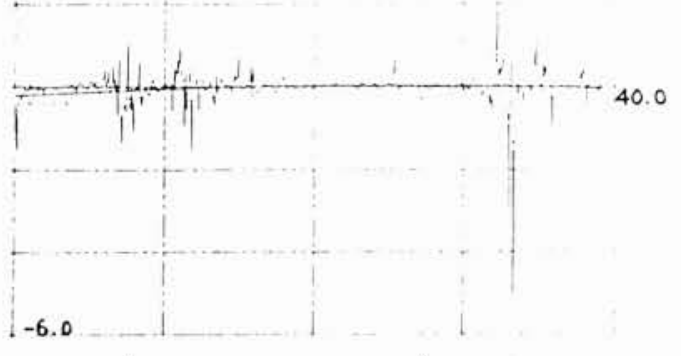

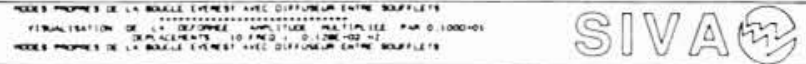

4 apput artatrectionnal $\mathrm{F}$.

- encastromat et cebit nut

- prewion nutie

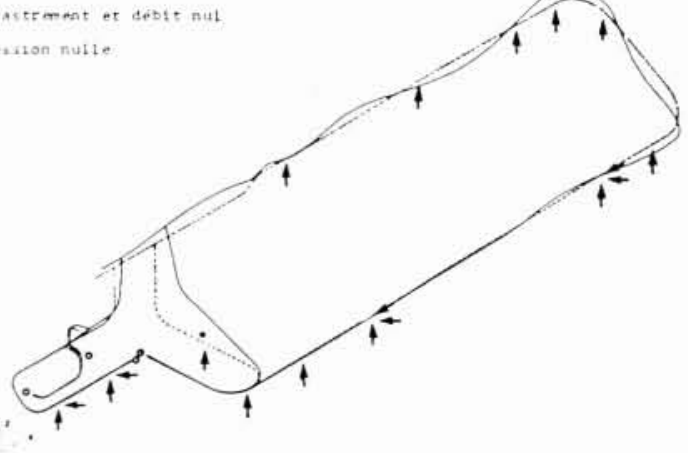

11 . Déformée modale à $13 \mathrm{~Hz}$.

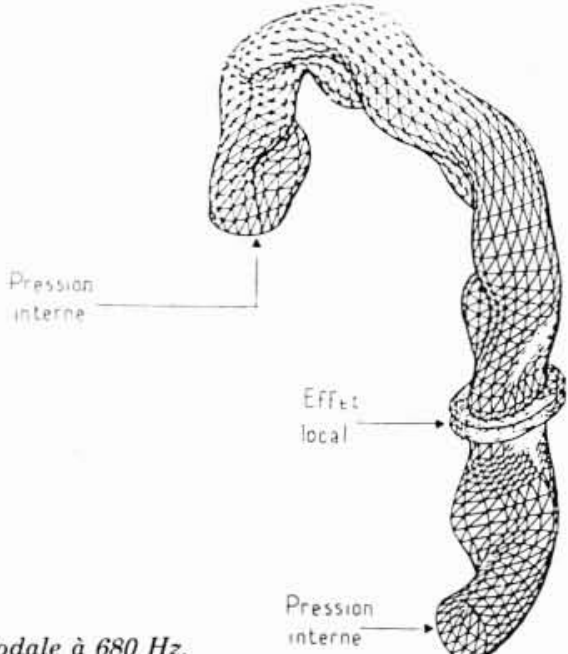

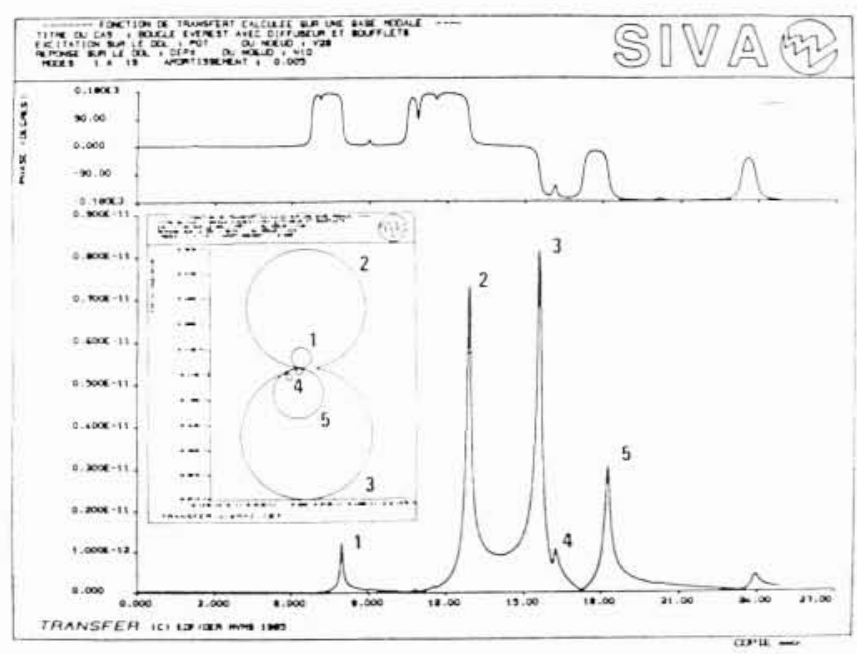

12. Fonction de transfert $\Delta P$ vanne-accélération axiale en diffuseur.

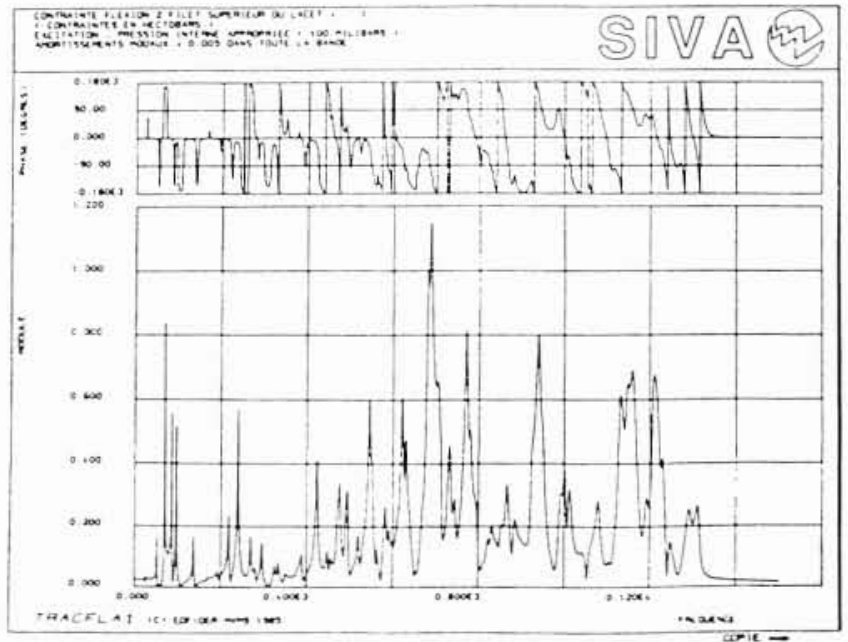

14. Fonction de transfert pression-contrainte. 
différents (T1: $10 \mathrm{~cm}$; T2: $20 \mathrm{~cm}$; T3: $35 \mathrm{~cm}$ ). Ces différents tronçons sont supportés ou fixés en plusieurs points (A), mais la boucle dans son ensemble garde une souplesse très importante en flexion.

\section{Identification d'une vanne}

Dans un premier temps, on examine la source associée à une vanne papillon (VI) qui participe de façon notable à l'excitation acoustique du circuit. Sa perte de charge est de l'ordre de 2 bar pour une vitesse d'écoulement de $5 \mathrm{~m} / \mathrm{s}$.

La méthode d'identification expérimentale est fondée sur l'analyse linéaire par matrice de transfert. Dans la mesure où la singularité à identifier n'est pas la seule du circuit, une série de 3 essais est nécessaire, pour lesquels on garde invariante la source recherchée (conservation de la géométrie et de l'écoulement dans la vanne) tandis que l'on fait varier les autres sources du circuit (pompes, vannes réglantes). La source identifiée est une discontinuité de pression dont la DSP présente une énergie plus importante dans les basses fréquences (cf. figure 9 ) et une structure assez comparable à celle d'une vanne à opercule (fréquence de coupure vers $20 \mathrm{~Hz}$ et niveau maximum de l'ordre de $1 \%$ de la perte de charge).

On a pu en outre obtenir les coefficients de la matrice de transfert du tube comportant la singularité (cf. figure 10 ).

\section{Calcul de la réponse}

On s'intéresse à présent à la réponse acoustique-mécanique de la boucle d'essai à l'excitation fluide que l'on a identifiée. Afin d'obtenir une configuration de couplage facile à valider, on a privilégié un type particulier de couplage, s'opérant par effet de fond dans un diffuseur conique.

Ce diffuseur est monté sur un tronçon droit (TD), introduit dans la veine par l'intermédiaire de deux soufflets de dilatation (S1 et S2). Deux appuis rigides (R1 et R2) encadrent les soufflets, et réalisent alors en principe l'isolation mécanique du diffuseur.

\section{Analyse des vibrations couplées}

On a élaboré un modèle d'éléments finis du système couplé décrit plus haut (tronçon souple comportant un diffuseur). On a calculé une quinzaine de résonances de l'ensemble de la boucle entre 0 et $25 \mathrm{~Hz}$, dont certaines mettent en jeu le couplage acoustique-mécanique au niveau du diffuseur (par exemple, figure 11). On a ensuite calculé la fonction de transfert entre la discontinuité de pression au niveau de la vanne $V_{1}$ et le déplacement axial du diffuseur. On y retrouve (figure 12) certaines des fréquences propres de la boucle:

Le pic 1 correspond à la résonance axiale du tronçon souple (raideur des soufflets, masse du tronçon et du fluide entraîné par le diffuseur). Le pic 4 est une résonance mécanique de l'ensemble de la boucle (le diffuseur est entraîné par le fluide, lui-même entraîné par la boucle). Le déplacement axial le plus important du diffuseur interviendra en fait aux résonances acoustiques de l'ensemble de la boucle : pour les pics 2 et 3 , on a $80 \%$ d'énergie vibratoire dans le fluide, tandis que le pic 5 correspond à une égale répartition entre le fluide et le tuyau. Il devient donc important, au-delà de cette dernière fréquence, de modéliser avec soin le comportement acoustique-mécanique de la boucle dans son ensemble, en particulier ses appuis.

Au stade du calcul des modes propres, on peut déjà conclure qu'il était intéressant a priori d'introduire un tronçon souple qui doit permettre à basse fréquence de s'affranchir de l'ajustement des conditions limites mécaniques des supportages de la boucle.

\section{Prévision des niveaux vibratoires}

En adoptant pour la vanne papillon une densité spectrale de puissance d'allure voisine de celle identifiée (dont le niveau maximum correspond à une pression RMS égale au centième de la recompression statique), on calcule à l'aide de la fonction de transfert décrite précédemment une valeur RMS de l'accélération axiale au niveau du diffuseur égale à $0,1 \mathrm{~g}$, ce qui correspondrait à une amplitude vibratoire de l'ordre de 10 microns à $15 \mathrm{~Hz}$.

\subsection{Vibration à moyenne fréquence d'une tuyauterie vapeur}

On a étudié le cas d'une tuyauterie industrielle véhiculant de la vapeur sous pression dans le cadre des phénomènes moyenne fréquence décrits plus haut. Plus précisément, on a cherché à évaluer en analysant des résultats de mesures (accélérations, contraintes et pression vapeur fluctuante) différents scénarii d'excitation pouvant justifier les phénomènes constatés.

\section{Modélisation du système}

On a calculé pour commencer les résonances mécaniques du tuyau entre 0 et $1200 \mathrm{~Hz}$ à l'aide d'un modèle d'éléments finis (figure 13). La densité modale est importante (environ 1 mode tous les $10 \mathrm{~Hz}$ ), et la plupart des résonances mettent en jeu la déformation de la coque. Le mode à $680 \mathrm{~Hz}$ de la figure illustre une telle déformation, qui participe à l'excitation des petites pièces situées sur la bride d'assemblage (B) comme le montre la fonction de transfert (figure 14) entre la contrainte de flexion dans l'un des goujons de bride, et un champ de pression interne dont la répartition spatiale est appropriée au déplacement radial de la coque (répartition qui est la plus conforme au scénario de couplage acoustique-mécanique). On a ensuite pu vérifier une corrélation correcte des niveaux moyens de contrainte et de pression fluctuante avec les niveaux mesurés. Cela justifie l'intérêt d'une hypothèse de sollicitation d'un élément mécanique par l'établissement d'une résonance acoustique, de la même façon que dans le cas présenté précédemment, qui était un cas basse fréquence.

\section{Prévision des dommages à moyenne fréquence}

$\mathrm{Si}$ on effectue une caractérisation expérimentale du champ de vitesse fluctuant tridimensionnel au voisinage de singularités particulières, (par exemple, par vélocimétrie laser), on peut en proposer une représentation simplifiée sous forme de spectres de sources acoustiques d'ondes non planes (qui s'étendront au-delà des fréquen- 
ces de coupure des ondes correspondantes). En supposant acquise une représentation plus ou moins précise en espace et en temps de ce type de source, on peut vouloir prédire la tenue en fatigue à moyenne fréquence de tuyauteries soumises à de telles sources. Le passage des paramètres statistiques des sources à ceux de la réponse va nécessiter l'intégration des fonctions de transfert dans toute la gamme des moyennes fréquences. Etant donnée la très grande densité des résonances acoustiques et mécaniques rencontrées en général dans cette gamme, on doit s'attendre à des coïncidences fréquentielles entre modes acoustiques et modes de coque. Or, les transferts d'énergie vibratoire entre le fluide et la coque interviennent dans des bandes de fréquence situées autour des coïncidences, d'autant plus étroites que les énergies vibratoires emmagasinées dans le fluide et le tuyau sont différentes (cas de tuyauteries véhiculant un fluide gazeux sous pression). Par conséquent, bien qu'il ne soit pas indispensable de recaler a priori chacune des résonances du fluide ou de la coque, il importe cependant d'évaluer correctement la densité fréquentielle de ces zones de transfert d'énergie.

Le modèle de poutre précédemment présenté est bien adapté au calcul d'un grand nombre de résonances acoustique-mécaniques dans un domaine fréquentiel étendu.

\section{Conclusion}

La prévision de la réponse vibratoire des circuits industriels aux sollicitations dues à l'écoulement du fluide est fondée sur une représentation aléatoire des sources indépendantes du reste du circuit (catalogue de singularités). L'identification des sources les plus simples est possible expérimentalement dans la mesure où la fonction de transfert du circuit entre le point source et le point de mesure de la réponse est bien connu. C'est le cas pour des configurations simples et à basse fréquence. On peut alors introduire une singularité particulière au sein d'un circuit quelconque et prévoir sa réponse.

Mais à des fréquences plus élevées, liées à des problèmes de bruit rayonné ou à des ruptures locales de petits assemblages, il faut disposer de fonctions de transfert plus complexes mettant en jeu la propagation dans le circuit d'ondes de coque et de fluide couplées entre elles. Un tel modèle de fonction de transfert a été proposé, bâti sur une extrapolation aux moyennes fréquences des modèles éléments finis de poutre valable à basse fréquence. Ce modèle permet de limiter le coût du calcul des modes propres qui sont généralement très nombreux aux moyennes fréquences.

\section{Références}

[1] Boulot F. and al. - Vibrations de structures dans un écoulement sous l'effet du détachement tourbillonnaire de sillage, Société hydrotechnique de France (1981).

[2] CHEN S.S. - Out-of-Plane vibration and stability of curved tubes conveying fluid, J. Applied Mech. 40, 362-368 (1973).

[3] BLEvins R.D. - Flow induced vibrations, Van Nostrand Reinhold Company (1977).

[4] CARTA F.O. - Unsteady gapwise periodicity of oscillating cascades airfoils (ASME $82-$ G.T. 286).
[5] Simpson H.C., Clarck T.A., Weir G.A. - A theoretical investigation of hydraulic noise in pumps, Journal of sound and vibration $1967-5$ (3) pp. 456-488.

[6] IMaichi K., Tsujimoto Y., Yoshida Y. - A 2-D analysis of the interaction effects of radial impeller in volute casing, AIRH Symposium 1980 - Tokyo.

[7] GiBERT R.J. - Sources d'excitation aléatoires, CEA Rapport DEMT/86/192.

[8] Garreau D. et TePhany F. - Efforts aérodynamiques instationnaires et vibrations des ailettes BP d'une turbine à vapeur. $R F M, \mathrm{n}^{\circ} 1986-3$.
M. le Président. - Quelle valeur d'amortissement prend-on dans le solide et dans le fluide?

M. GIBERT. - C'est une question importante : l'amortissement peut être dû à la structure mécanique, au fluide, mais il trouve parfois son origine dans l'hydroélasticité, c'est-à-dire le mécanisme de couplage entre structure et fluide en écoulement. Dans la plupart des cas, l'écoulement augmente les taux d'amortissement.

En pratique, on travaille avec des valeurs empiriques. Ainsi, on a tendance à prendre une valeur de 1 à $3 \%$ pour une tuyauterie en écoulement. Mais il faut se méfier des cas particuliers où l'on observe l'inverse et pour lesquels, à partir d'une certaine vitesse d'écoulement, le système devient instable.

M. THIRRIOT. - Vous avez surtout parlé des régimes permanents; en ce qui concerne les transitoires, doit-on les ranger dans la partie acoustique?
M. GIBERT. - A partir du moment où l'on peut caractériser ces transitoires par des lois linéaires, ce sont les mêmes équations. Dans le cas des coups de bélier, l'hypothèse de linéarité est souvent bonne.

M. le Président. - Avez-vous en tête des exemples autres que les coups de bélier où l'on ne linéarise pas tout?

M. GIBERT. - Dans les tuyauteries, certains effets sont vraiment non-linéaires. Je pense aux phénomènes de fouettement pour lesquels l'entrainement du fluide est important et induit des efforts non-linéaires sur une structure elle-même non-linéaire. En calcul numérique, l'approche linéaire est traitée par le modèle acoustique qui a été présenté; le cas non linéaire nécessite l'emploi de méthodes d'intégration temporelle souvent explicite utilisant une formulation du type Euler-La grange: on traite ainsi des grands déplacements. 